\title{
Erinnerungen an das Rostocker Kunstherz
}

Das 18. Dresdner Symposium konnte einen prominenten Rostocker Kardiologen begrüßen, der noch zu DDRZeiten an einem totalen Herzersatz arbeitete: Prof. W. Urbascek. Einen Prototyp spendierte Urbascek der Medizinhistorischen Sammlung der Deutschen Gesellschaft für Kardiologie (DGK). In einer Ausstellung wurde u.a. die Neuerwerbungen der Sammlung gezeigt, darunter auch das "Rostocker TAH-Modell, eine Dauerleihgabe des Instituts für Biomedizinische Technik der Universität Rostock."

Dass Prof. Urbascek persönlich anwesend war und zu seiner Entwicklungsarbeit befragt werden konnte, war geradezu eine medizinhistorische Sternstunde. 1981 erschienen in Langen- becks Archiv für Chirurgie (Volume 355, Issue 1, S. 649) „Erste Erfahrungen mit dem Rostocker künstlichen Herz." In der Zusammenfassung der Arbeit hieß es: „Das Programm des Rostocker künstlichen Herzens (TAH) konzentriert sich hauptsächlich auf Biomaterialien und mechanische Eigenschaften und Anpassung und Formgebung des TAH für den zukünftigen Einsatz am Menschen. Die Brauchbarkeit des TAH wurde tierexperimentell überprüft, wobei die chirurgische Technik von der Arbeitsgruppe in Utah (USA) übernommen wurde. Bei Anpassungsversuchen an 23 Verstorbenen wurden verschiedene Herztypen und ihre unterschiedliche Lokalisation überprüft."

(J.A.) I

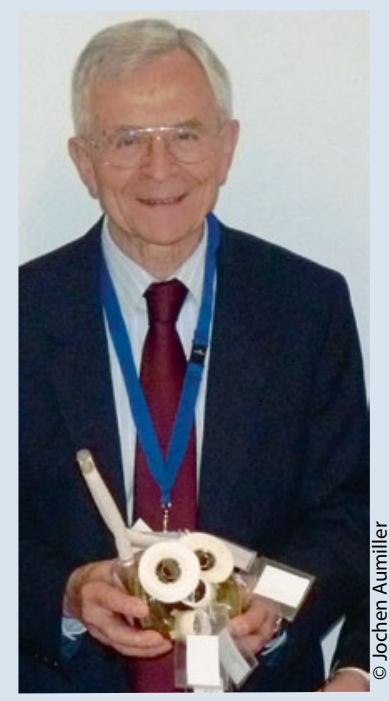

Prof. Urbascek mit Rostocker Kunstherz.

\section{Gespräch mit dem neuen Lipid-Liga-Vorsitzenden, Ulrich Laufs, Homburg / Saar und seinem Vorgänger Achim Weizel, Mannheim}

\section{„Statine sind unsere stärkste Waffe}

\author{
„Nur mit Statinen gelingt es, \\ das kardiovaskuläre Risiko in \\ der primären und sekundär- \\ en Prävention zu verringern." \\ Prof. Achim Weizel, Mannheim \\ ehemaliger Vorsitzender der Lipid-Liga
}
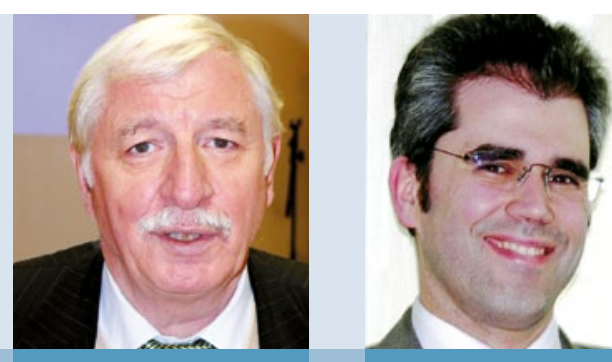

„Das LDL-Cholesterin ist der entscheidende Risikofaktor. Hier gilt nach wie vor: the lower the better."

Prof. Ulrich Laufs, Homburg/Saar, neuer Vorsitzender der Lipid-Liga
Die deutsche Lipid-Szene ist im Umbruch: Mit Prof. Ulrich Laufs hat die Lipid-Gesellschaft einen neuen Vorsitzenden, zum anderen stehen altbekannte Dogmen infrage und vor allem gibt es neue vielversprechende Forschungsansätze zur Therapie der Dyslipidämien. Folgendes Gespräch entstand im Anschluss an den 5. Deutschen Atherosklerosekongress im Dezember 2012 in München.
CardioVasc: Prof. Weizel, in Ihrer Zeit als Liga-Präsident fällt die Etablierung der Statine als antiatherosklerotische Basismedikation und als Therapie von Dyslipidämien: Statine sind sozusagen das Aspirin für den Fettstoffwechsel. Dennoch bleibt ein Restrisiko. Werden Statine nicht überschätzt?

Weizel: Im Gegenteil, nur mit den Statinen gelingt es, das kardiovaskuläre Risiko in der primären und sekundären Prävention zu ver- ringern. Die Beeinflussung von Risikofaktoren wie Geschlecht, Alter, Tabakkonsum, Übergewicht ist schwierig, gar nicht möglich oder in der Effektivität den Statinen unterlegen. Die Statine sind die stärkste Waffe, sie werden auch zukünftig die Basistherapie sein. Denkbar ist jedoch, dass Medikamente mit neuen Wirkprinzipien zusätzlich eingesetzt werden.

CardioVasc: Ein Paradigmenwechsel in Ihrer Zeit war die Risikostratifizierung. Die 